\title{
Non-contact electrochemical evaluation of biofilms
}

\author{
Charles E. Turick $^{1}\left[\right.$ D Hector Colon-Mercado ${ }^{2} \cdot$ Christopher E. Bagwell $^{3} \cdot$ Scott D. Greenway ${ }^{4} \cdot$ Jake W. Amoroso $^{5}$
}

Received: 11 November 2019 / Accepted: 21 January 2020 / Published online: 12 February 2020

(C) The Author(s) 2020 OPEN

\begin{abstract}
Here, we demonstrate a non-contact technique for electrochemical evaluation of biofilms on surfaces in relation to corrosion. Electrochemical impedance spectrometry was employed, incorporating flat patterned electrodes positioned over the surfaces of aluminum and glass with and without biofilms. Signal communication from the working electrode to the counter electrode followed electric field lines passing tangentially through the biofilms. Electrochemical impedance parameters that were evaluated included complex impedance, phase angle, imaginary (out of phase) conductivity and Cole-Cole plots with a corresponding equivalent circuit. Changes in the impedance properties due to the presence of biofilms were monitored and correlated through microbiological, chemical and electrochemical assays. Impedance parameters associated with microbial activity correlated with biofilms on aluminum and glass surfaces. This technical approach provides impedance information about the biofilm without the signal traveling through the underlying conductive media or disrupting the biofilm. In this way, biological contributions to surface fouling can be evaluated with minimal contribution from the inorganic surface under the biofilm. In addition, this technique can be used to monitor biofilms on electrochemically inert surfaces as well as electrically conductive surfaces.
\end{abstract}

\section{Graphic abstract}

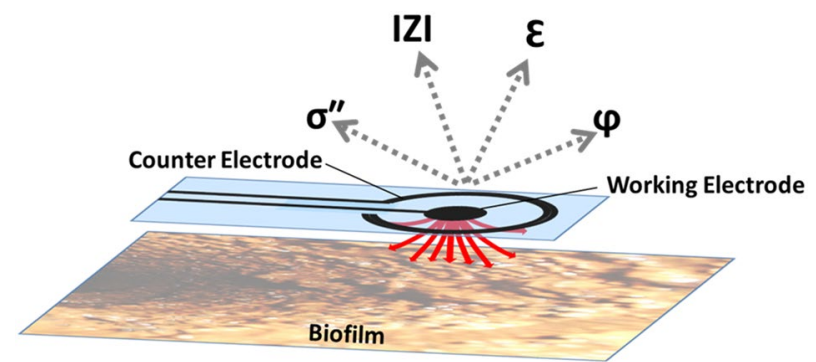

Keywords Electrochemical impedance spectroscopy · Biofilm · Microbially induced corrosion · Potentiodynamic characterization · Electrochemically inert surfaces

Charles E. Turick, Charles.Turick@srnl.doe.gov; Hector Colon-Mercado, Hector.Colon-Mercado@srnl.doe.gov; Christopher E. Bagwell, Christopher.Bagwell@pnnl.gov; Scott D. Greenway, Scott@greenway-energy.com; Jake W. Amoroso, Jake.Amoroso@srs.gov|

${ }^{1}$ Environmental Sciences and Biotechnology, Savannah River National Laboratory, Building 999-W, Aiken, SC, USA. ${ }^{2}$ Renewable Energy Programs, Savannah River National Laboratory, Building 999-2 W, Aiken, SC, USA. ${ }^{3}$ Earth Systems Science, Pacific Northwest National Laboratory, PO Box 999, Richland, WA, USA. ${ }^{4}$ Greenway Energy LLC, 301 Gateway Dr., Aiken, SC, USA. ${ }^{5}$ Engineering Process Development, Savannah River National Laboratory, Building 999-W, Aiken, SC, USA. 


\section{Introduction}

Microbially influenced corrosion (MIC) can significantly deviate from predictable changes that metals would otherwise undergo during classically defined inorganic corrosion [1] because biofilms induce changes to electrochemical conditions at the metal/solution interface. These changes are difficult to predict due to variations of microbial populations and growth conditions from one location to another. Numerous techniques have been applied to studying MIC. Especially useful are electrochemical techniques for evaluation of corrosion effects on materials and include measurement of redox potential or corrosion potential, electrochemical noise analyses, electrochemical impedance spectrometry (EIS), polarization resistance and cyclic voltammetry [2]. MIC is typically investigated using conventional electrochemical techniques to measure the current and voltage applied directly to the surface or techniques like pitting scans and potentiostatic and potentiodynamic polarization curves to provide useful mechanistic information on corrosion parameters. These methods generally cause irreversible changes to the surfaces from physical contact and large-signal polarizations. A technique that preserves the integrity of the biofilm would broaden the available options for suppressing and detecting MIC. Recently, Tanaka et al. [3] described a non-contact method for the in situ detection of biofilms through the determination of biofilm hydrophobicity, which offers potential in determining surface changes for the development of antimicrobial materials. In this work, we report a non-contact EIS method using commercially available patterned electrodes to measure MIC on surfaces.

This work was motivated by the concept that a fielddeployable, in situ EIS evaluation of MIC could provide a remote, non-contact method to aid in determining the status of systems undergoing MIC. As an example, commercial used nuclear fuel is stored under water in cooling basins until its radioactivity is low enough for dry storage [4], permanent disposal or reprocessing [5]. Aluminum clad fuel makes up a significant portion of the fuel in storage across the USA, and the presence of MIC on the integrity of the fuel cladding material is of particular interest considering that the fuel has or is expected to exceed its anticipated time spent in storage [6-8]. Despite the low nutrient availability of the purified water maintained in nuclear cooling pools, microbial activity has been reported along with biofilm formation and MIC [5-9] and is associated with diverse bacterial phyla including Proteobacteria, Nitrospirae, Deinococcus-Thermus, Sphingobacteria, Actinobacteria and
Planctomyces [9]. The growth and survival of mixed species of typical air-borne soil bacteria as biofilms on cladding material exposed to $2 \mathrm{~Gy} / \mathrm{h}$ (total dose $4.9 \times 10^{3} \mathrm{~Gy}$ ) [8] demonstrate that radiation does not prevent biofilm formation in these environments. More recent work indicates that storage basins are complex, dynamic systems, in which chemical, biological and physical conditions vary spatially and evolve temporally, thereby demonstrating a need to reevaluate corrosion surveillance programs in nuclear storage basins [9].

Cooling water in nuclear storage basins is typically monitored for microbial activity to provide some measure of the probability of MIC formation on cladding material. Although aquatic microbial populations that are suspended in the water are not a good indicator of nearby biofilm development and activity $[1,10]$ including on cladding material [7], at present it is logistically impractical to monitor fuel assemblies directly for biofilm activity.

Electrochemical techniques, including EIS, provide useful information regarding metal corrosion behavior and associated biofilm activity on surfaces. Microbial biofilms in the subsurface have been monitored previously with electrochemical impedance techniques [11-13] without physically contacting the biofilms. Impedance analysis of biofilms provides a data-rich platform with parameters associated with microbial activity. For instance, the complex impedance (IZI) and phase angle $(\varphi)$ are influenced by lipid membranes $[14,15]$. In addition, imaginary (out of phase) conductivity $\left(\sigma^{\prime \prime}\right)$ and dielectric permittivity $(\varepsilon)$ are associated with biofilm formation $[11,13]$ and microbial activity $[16,17]$. Hence, bacterial biofilms influence electric double-layer interactions on the surfaces to which they are attached [18] and have an effect on double-layer capacitance as determined with EIS [19, 20].

As a result, a non-contact approach to monitor mature biofilms on aluminum surfaces was investigated. To avoid surface contact, flat patterned electrodes were positioned at a predetermined distance parallel to biofilm surfaces. Signal communication from the working electrode (WE) to the counter electrode (CE) followed electric field lines passing tangentially [21] through the biofilms. Changes in the impedance properties due to the presence of biofilms were monitored and correlated with MIC.

\section{Materials and methods}

\subsection{Microbial cultures}

Water samples from near the top surface of aluminum fuel rod storage canisters (Savannah River Site, Aiken, SC) were obtained and stored at $4{ }^{\circ} \mathrm{C}$ for $24 \mathrm{~h}$ prior to microbiological characterization. Pure cultures were isolated with 
conventional culturing methods by streaking to isolation on R2A plating medium. Pure cultures were then maintained on tryptic soy agar (TSA) or broth (TSB).

Pure colonies were suspended in a 5\% Chelex 100 resin solution and boiled for $10 \mathrm{~min}$ to lyse bacterial cells. Cell debris was pelleted by centrifugation ( $6000 \times \mathrm{g}, 1 \mathrm{~min})$, and DNA quality and quantity were evaluated using a NanoDrop spectrophotometer. The $16 \mathrm{~S}$ ribosomal RNA subunit gene was PCR-amplified using universal bacterial primers (27G/1492R, E. coli numbering) and conventional thermal cycling. Amplification accuracy and specificity were assessed by gel electrophoresis, and the products were purified by alcohol precipitation in high salt. The purified PCR products were suspended in $10 \mu \mathrm{L}$ of $\mathrm{ddH}_{2} \mathrm{O}$ for DNA sequencing. The sequences were trimmed to remove weak and ambiguous base calls in Sequencher (v 4.8), and the remaining $>400$ bp partial rrn gene sequences were queried against the NCBI and RDP databases for putative identification. All IDs were confirmed by having $>99 \%$ sequence identity to authentic gene sequences available in both sequence databases. Three cultures dominated the sample and were rod-shaped, mesophilic heterotrophs identified as a Gram-negative, non-spore-forming facultative anaerobe, Serratia marcescens; a Gram-negative, non-spore-forming aerobe, Delftia acidovorans; and a Gram-positive, facultative anaerobic spore former, Bacillus thuringiensis.

\subsection{Biofilm growth}

For proof of principle that this technique can be used to detect and monitor biofilms, glass slides were chosen as an inert surface. Shewanella oneidensis MR-1, a well-characterized bacterium often associated with biofilms and corrosion, was chosen in this portion of the study. Cultures were grown in $50 \mathrm{~mL}$ of TSB in the presence of clean, sterile glass slides for 1 week at $28^{\circ} \mathrm{C}$. The duplicate cultures produced a confluent biofilm on the glass surfaces (as determined with light microscopy) resulting in $16.1 \mu \mathrm{g} / \mathrm{cm}^{2}( \pm 2.4 \mu \mathrm{g} /$ $\mathrm{cm}^{2}$ ) of bacterial protein.

Cultures of $S$. marcescens, D. acidovorans and B. thuringiensis were grown in TSB, in triplicate, at $28^{\circ} \mathrm{C}$ for $24 \mathrm{~h}$ followed by inoculation (10\%/vol) into $10 \%$ TSB as described previously [8] in the presence of sterile aluminum coupons. Coupons were individually numbered (engraved) on the one side with incubation of the coupons occurring horizontally, with the numbered side down. The cultures and sterile controls were maintained for 6 weeks with the medium refreshed weekly.

Coupons of 1100 aluminum alloy $(99.0 \% \mathrm{Al})$ were chosen because this alloy is identical to the cladding surface of many types of fuels and fuel storage materials contained in the U.S. DOE, Savannah River Site storage facilities. The coupons were $4.0 \mathrm{~cm}$ in diameter and produced by centerless grinding (Metal Samples Co., Munford, AL). Prior to analysis, all coupons (with or without biofilms) were rinsed in sterile $50 \mathrm{mM}$ phosphate-buffered saline (PBS) three consecutive times to remove unattached bacteria [22].

\subsection{Electrochemical analyses}

\subsubsection{Instrumentation}

All electrochemical studies were performed on a fourchannel potentiostat, VersaSTAT MC (Princeton Applied Research, Princeton, NJ) with data recorded via VersaStudio software version 2.42.3.

2.3.1.1 Electrode configuration Flat patterned electrodes (Pine Instruments, Grove City, PA) consisted of 2-mmdiameter graphite WE surrounded on three sides by a graphite $\mathrm{CE}$ with an $\mathrm{Ag} / \mathrm{AgCl}$ reference electrode. Electrodes were fitted to a solid support (glass slide) to provide extra rigidity. With the electrodes facing out, they were positioned above and parallel to surfaces for noncontact evaluation of surface biofilms using 3-mm-thick
Fig. 1 Image of flat patterned electrode and schematic of electrode configuration for non-contact biofilm analysis. Flat patterned electrode was positioned parallel to the surface of interest. Surface biofilm was probed during communication between working and counter electrodes. The electrode was positioned $3 \mathrm{~mm}$ from the surface being analyzed using chemically inert septa placed so as to not interrupt the signal to the counter electrode

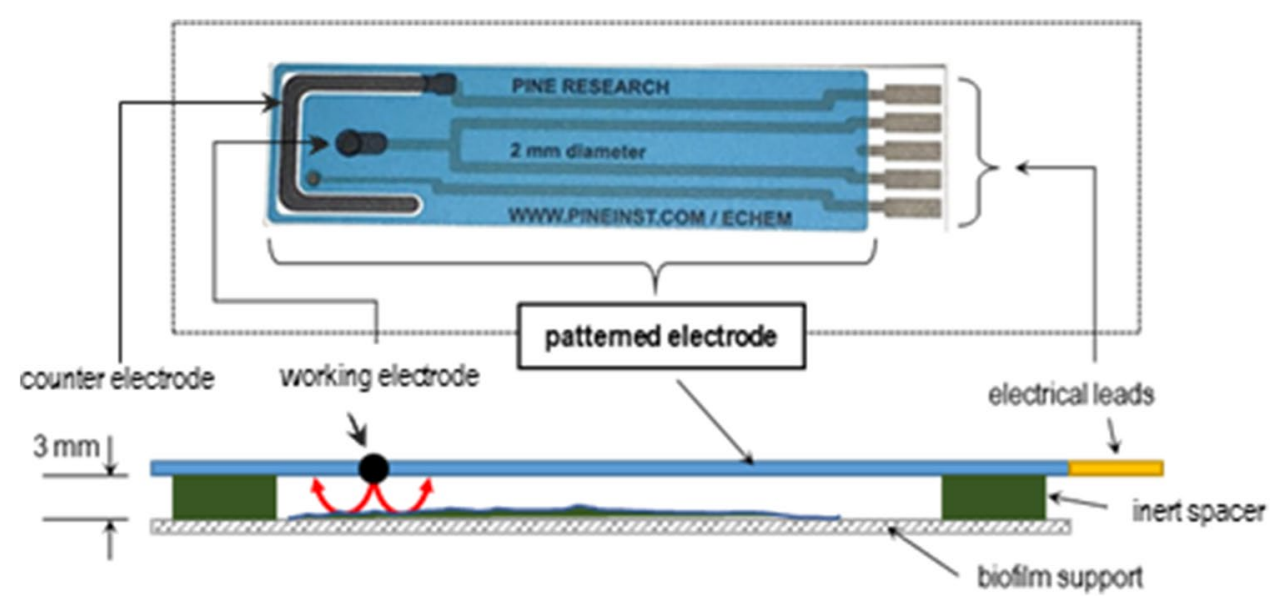

SN Applied Sciences A SPRINGER NATURE journal 
chemically inert septa (Reztech, Tempe, AZ) (Fig. 1). The septa were arranged to maintain a constant distance between surfaces but not to interfere with signals. Signal communication from the WE to the CE followed electric field lines passing tangentially over surfaces studied, including biofilms [21].

\subsubsection{EIS analysis}

Immediately prior to EIS analysis, all coupons (with or without biofilms) were rinsed in sterile $50 \mathrm{mM}$ phosphatebuffered saline (PBS) three consecutive times to remove suspended bacteria not part of a biofilm. All EIS analyses were conducted in PBS to minimize any effects from the diffusion of microbial metabolites. EIS measurements were carried out at open-circuit potential with a sinusoidal signal perturbation of $50 \mathrm{mV}$, with a frequency range of $10^{5}-10^{-2} \mathrm{~Hz}$. Ten measurements were recorded per decade of frequency with a measurement delay of $2 \mathrm{~s}$. Data were analyzed with ZView software (Scribner Associates, Southern Pines, NC). EIS was performed immediately following three cycles of voltammetric stripping and conditioning of the electrode $(-2$ to $+2 \mathrm{~V}: 1 \mathrm{~V} / \mathrm{s})$. Data were evaluated as Bode plots, i.e., phase $(\varphi)$ plots. Imaginary conductivity $\left(\sigma^{\prime \prime}\right)$ was also determined where $\sigma^{\prime \prime}$ is defined as

$\sigma^{\prime \prime}=Y^{\prime \prime} \frac{d}{a}$

with $Y^{\prime \prime}$ defined as imaginary admittance:

$Y^{\prime \prime}=\frac{-Z^{\prime \prime}}{Z^{\prime 2}+Z^{\prime \prime 2}}$

where $d=$ distance between the electrodes and $a=$ effective area of the electrode.

The complex dielectric function [13] was presented as Cole-Cole plots where real $\left(\varepsilon^{\prime}\right)$ permittivity and imaginary $\left(\varepsilon^{\prime \prime}\right)$ permittivity are defined as follows:

$\varepsilon=\frac{-Z^{\prime \prime}}{\left(Z^{\prime 2}+Z^{2 \prime \prime}\right) \omega C_{0}}$

$\varepsilon^{\prime \prime}=\frac{-Z^{\prime}}{\left(Z^{\prime 2}+Z^{\prime \prime 2}\right) \omega C_{0}}$

where $\omega=$ angular frequency and $C_{0}=$ capacitance of an empty cell.

\subsubsection{Potentiodynamic characterization}

Electrochemical polarization (Tafel) curves were performed by scanning the potential from -800 to $-400 \mathrm{mV}$ with $\mathrm{Ag} / \mathrm{AgCl}$ reference with identical, clean Al 1100 coupons as counter electrodes with a sweep rate of $1 \mathrm{mV} / \mathrm{s}$.

\subsection{Microscopic analysis}

Confirmation of biofilms on surfaces was determined by acridine orange staining using an epifluorescence microscope [23].

Scanning electron microscopy (SEM) and energy-dispersive X-ray spectroscopy (EDS) measurements were performed using a Hitachi TM3000 SEM equipped with a Bruker Quantax EDS detector. The samples were mounted on an aluminum stub with carbon tape and were not coated. The instrument uses an automatically controlled backscattered detector to achieve topographical information in addition to the phase-contrast information. The EDS detector was calibrated with Cu standard prior to measurements.

\subsection{Biofilm removal and analysis}

Following EIS analysis, surfaces with biofilms and Al coupons (controls) were placed in sterile $100-\mathrm{mL}$ beakers and sonicated individually for $30 \mathrm{~min}$ in $50 \mathrm{~mL}$ of sterile PBS. The biofilm-free Al coupons were then rinsed and used in potentiodynamic studies. The dislodged biofilms were then concentrated via centrifugation and assayed for protein. A portion of biomass from Al coupons was prepared for Al determinations using EDS.

\subsection{Protein determinations}

Sonicated, pelleted cells were re-suspended in $800 \mu \mathrm{L}$ of PBS for protein measurement with DC Protein Assay (BioRad Cat. \# 500-0112) to quantify biofilm densities on Al coupons. Reagents were prepared as described by the kit manual. Colorimetric changes were measured using a Synergy H4 Multi-Mode Reader (BioTek). Data were analyzed using Gen5 Data Analysis Software (BioTek) and plotted in Microsoft Excel.

\section{Results}

\subsection{EIS analysis of bacterial biofilms on glass}

Seven-day-old confluent biofilms of S. oneidensis MR-1 on glass slides contained $16.1 \mu \mathrm{g} / \mathrm{cm}^{2}\left( \pm 2.4 \mu \mathrm{g} / \mathrm{cm}^{2}\right)$ of bacterial protein when EIS analysis occurred. EIS analysis was conducted with flat patterned electrodes without physically contacting the biofilm. Figure 2 demonstrates the comparisons of the rinsed biofilms in $50 \mathrm{mM}$ PBS to that of clean glass slides. The biofilms demonstrated a decrease in overall impedance (IZI) below $2 \mathrm{kHz}$ (Fig. 2a). The increase in biomass on the slides (relative to pristine glass) resulted in a phase shift (Fig. 2b). Background subtraction of the 

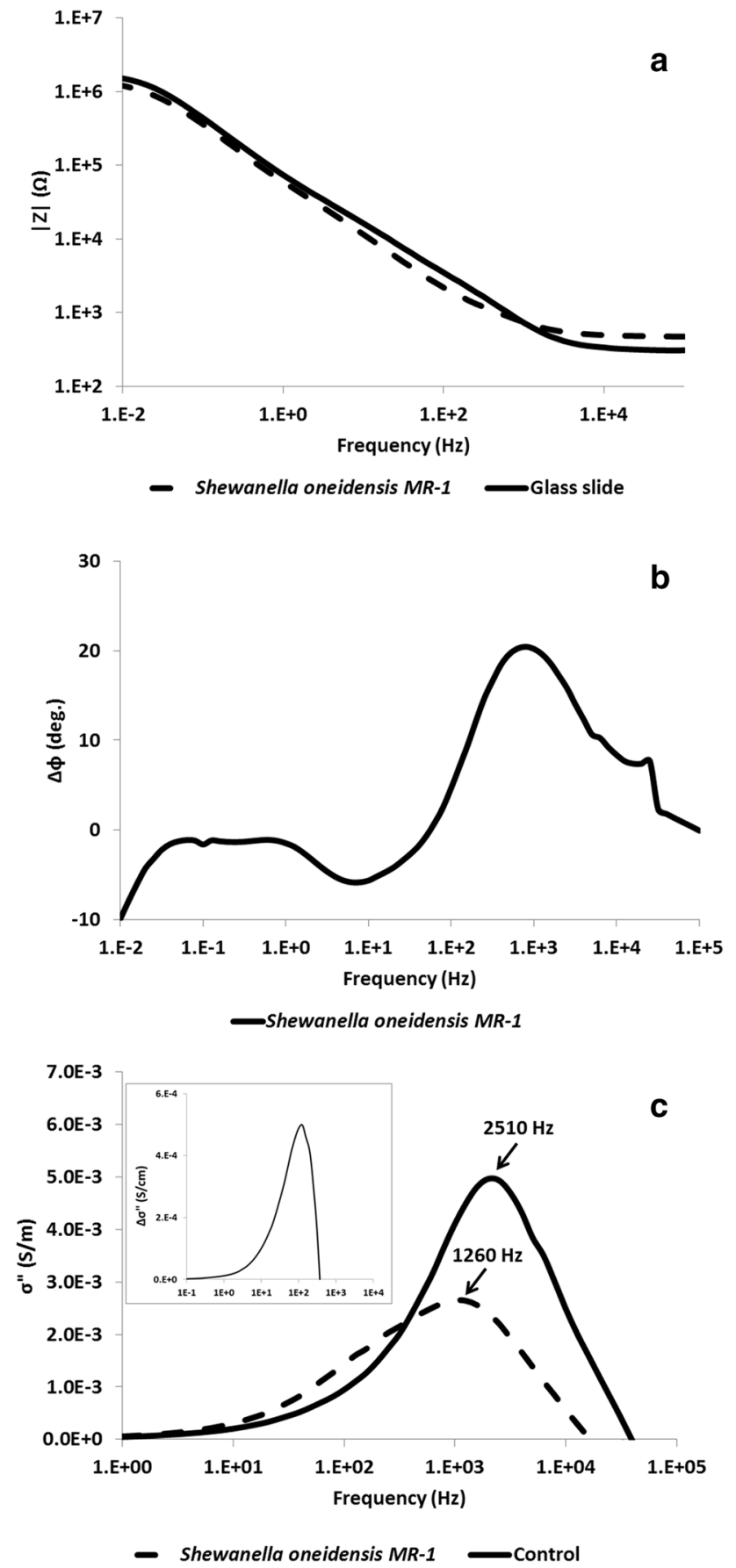

Fig. 2 EIS parameters of S. oneidensis MR-1 biofilms on glass. Biofilms on glass and glass slides alone were analyzed for IZI (a), phase shift relative to sterile glass controls $(\Delta \varphi)(\mathbf{b})$ and imaginary conductivity $\left(\sigma^{\prime \prime}\right)(\mathbf{c})$ with a relative difference $\left(\Delta \sigma^{\prime \prime}\right)$ between control and biofilm, below about $0.5 \mathrm{kHz}$ (inset)

data from the pristine glass control $(\Delta \varphi)$ showed a peak at $1 \mathrm{kHz}$ and is indicative of the increased presence of lipid membranes $[14,15]$. The imaginary conductivity $\left(\sigma^{\prime \prime}\right)$ of the biofilms exhibited a peak at $1.26 \mathrm{kHz}$ compared to $2.51 \mathrm{kHz}$, which was measured under control conditions
(Fig. 2c), and is consistent with the presence of attached biofilms, with increased $\Delta \sigma^{\prime \prime}$ indicative of increased microbial biomass [11-13].

\subsection{EIS analysis of bacterial biofilms on aluminum coupons}

Rinsed aluminum coupons containing 42-day-old biofilms were compared to sterile, clean aluminum coupons using EIS. Biofilms were determined by AO staining and fluorescence microscopy (Fig. 3). The degree of biofilm growth of the three pure cultures varied throughout the experiments (Table 1) with heterogeneous thickness calculated via confocal microscopy and ranging from 5 to $20 \mu \mathrm{m}$. Cultures of $S$. marcescens and D. acidovorans demonstrated the greatest amount of biofilm formation (Table 1). The Bode plot of biofilms from these two cultures exhibited decreased impedance at frequencies lower than $10 \mathrm{kHz}$, relative to sterile control coupons (Fig. $4 a$, b). This was similar to S. oneidensis MR-1 data on glass (Fig. 2). When the biofilms on aluminum were removed through sonication, impedance values were restored back to those of controls or somewhat higher, indicating that biofilms played a significant role in lowering the impedance of the aluminum coupons. $B$. thuringiensis was detected at relatively low biofilm density on coupons (Table 1), and there is no detectable difference in overall impedance relative to controls (Fig. 4c). Removal of biofilms from this culture (through sonication) did not change impedance values.

S. marcescens and D. acidovorans cultures on aluminum coupons also influenced the phase angle between $10^{5}$ and $10^{2} \mathrm{~Hz}$ (Fig. $\left.5 \mathrm{a}, \mathrm{b}\right)$ and were similar to that of S. oneidensis MR-1 on glass (Fig. 2b). The minimal growth of $B$. thuringiensis, however, provided a minimal contribution to $\Delta \varphi$ (Fig. 5c). A slight increase in the peak height of $\Delta \varphi$ was also seen between 10 and $0.1 \mathrm{~Hz}$ with $\mathrm{S}$. marcescens and $D$. acidovorans on aluminum (Fig. $5 \mathrm{a}$, b) but was not detected with biofilms on glass (Fig. 2b). The presence of aluminum associated with cellular biomass (Table 1) and the ability of aluminum oxides to effect $\varphi$ between 10 and $0.1 \mathrm{~Hz}$ [24] prompted the evaluation of aluminum oxide with our technique. Figure 6 demonstrates $\Delta \varphi$ of $\mathrm{Al}_{2} \mathrm{O}_{3}$ on glass with increased $\Delta \varphi$ between 10 and $0.1 \mathrm{~Hz}$, suggesting that $\mathrm{Al}_{2} \mathrm{O}_{3}$ associated with biofilms could contribute to $\Delta \varphi$ at the lower frequencies.

Biofilms of S. marcescens and D. acidovorans were associated with $\sigma^{\prime \prime}$ peak values that were shifted to lower frequencies by over $1 \mathrm{kHz}$ and $2 \mathrm{kHz}$, respectively (Fig. 7a, b). The sonicated coupons had similar $\sigma^{\prime \prime}$ peak values to that of controls (Fig. 7a, b). The $\sigma^{\prime \prime}$ peak value for $D$. acidovorans was higher than controls and likely indicates a biofilmassociated chemical change to the coupon (Fig. 7b). The low biofilm density of $B$. thuringiensis did not demonstrate 

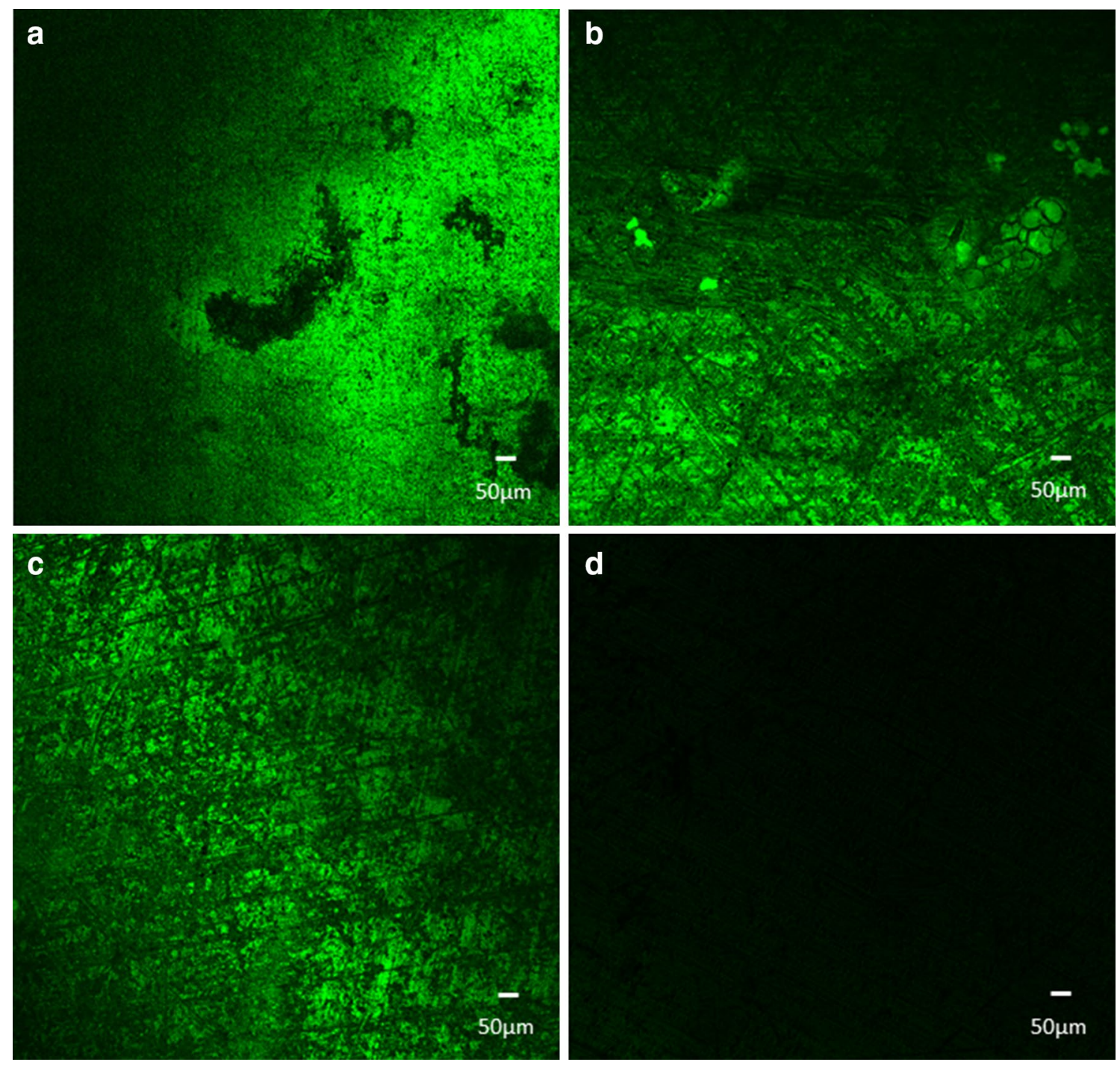

Fig. 3 Micrographs obtained under identical conditions demonstrated the presence of heterogenous bacterial biofilms on alu-

Biofilms fluoresce green with this technique. a S. marcescens; b $D$. acidovorans; $\mathbf{c} B$. thuringiensis; d abiotic control (no biofilm). Scale bars are shown at the lower right of each panel

Table 1 Biofilm evaluation from aluminum coupons

\begin{tabular}{lllll}
\hline Treatment & Cellular protein $\left(\mu \mathrm{g} / \mathrm{cm}^{2}\right)$ & $E_{\text {ocp }}(E \mathrm{vs} \mathrm{Ag} / \mathrm{AgCl})$ & $I_{\mathrm{o}}\left(A \times 10^{-6}\right)$ & $\mathrm{Al}(\%$ of biomass $)$ \\
\hline Sterile aluminum & Not detected & -0.628 & 3.80 & Not detected \\
D. acidovorans & $1.2 \pm 0.08$ & -0.660 & 1.62 & $6.2 \pm 0.9$ \\
B. thuringiensis & $0.3 \pm 0.01$ & -0.603 & 3.55 & $17.9 \pm 2.6$ \\
S. marcescens & $11.7 \pm 0.23$ & -0.670 & 4.78 & $9.6 \pm 1.2$ \\
\hline
\end{tabular}

any changes to $\sigma^{\prime \prime}$ peak values before or after sonication (data not shown). Increased values for $\Delta \sigma^{\prime \prime}$ are indicative of increased microbial biomass [11-13].

Permittivity values were reported as Cole-Cole plots and modeled with the circuit in Fig. 8. The presence of biofilms on glass demonstrated differences in permittivity relative to sterile controls at the lower frequencies
(Fig. 9a). At the higher frequencies, biofilms demonstrated increased double-layer capacitance, based on the increase in diameter of the semicircle (Fig. 9a, inset). At the lower frequencies, the Cole-Cole plot of biofilm on glass corresponds to the diffusion of the signal into the biofilm as opposed to the limited diffusion exhibited by the glass only (Fig. 9a). Cole-Cole

\section{SN Applied Sciences}



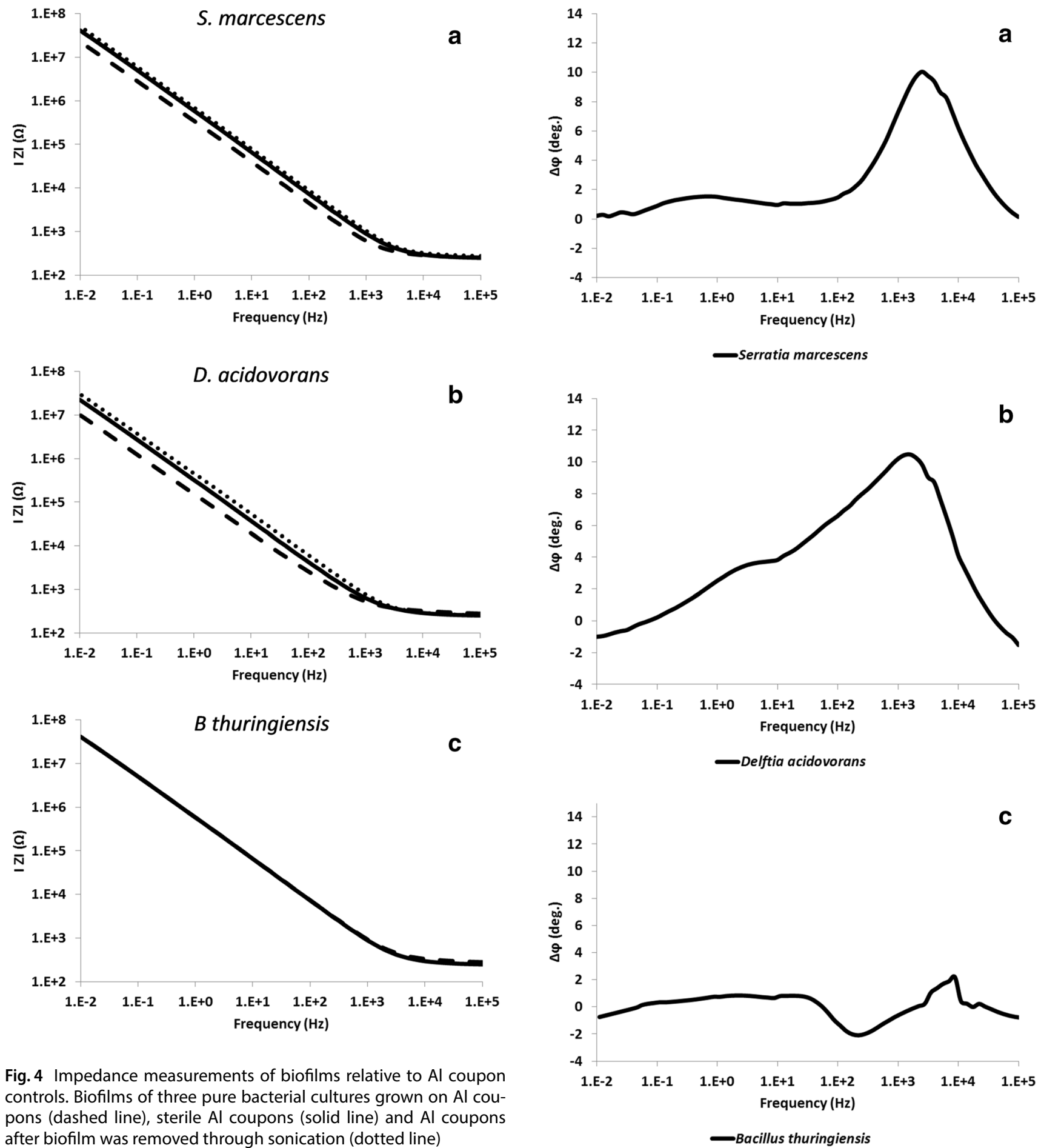

Fig. 4 Impedance measurements of biofilms relative to Al coupon controls. Biofilms of three pure bacterial cultures grown on Al coupons (dashed line), sterile Al coupons (solid line) and Al coupons after biofilm was removed through sonication (dotted line)

Fig. 5 Phase shift $(\varphi)$ of biofilms on Al coupon with sterile controls subtracted

plots demonstrated a greater difference in permittivity between biofilms on Al coupons and sterile controls (Fig. 9b). Overall permittivity associated with biofilms of S. marcescens and D. acidovorans cultures was diminished compared to sterile aluminum controls.

\subsection{Potentiodynamic analysis}

Biofilms produced by the three cultures grown on aluminum demonstrated changes to exchange currents $\left(I_{0}\right)$ compared to sterile controls (Fig. 10 and Table 1) with $D$. 


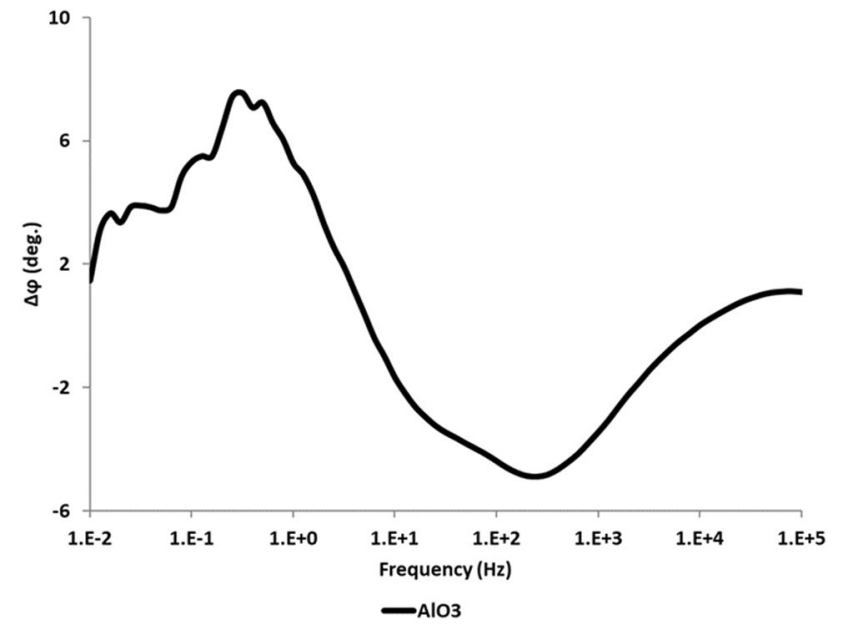

Fig. 6 Relative phase shift $(\Delta \varphi)$ of $\mathrm{Al}_{2} \mathrm{O}_{3}$ on glass (with background from glass slide subtracted)
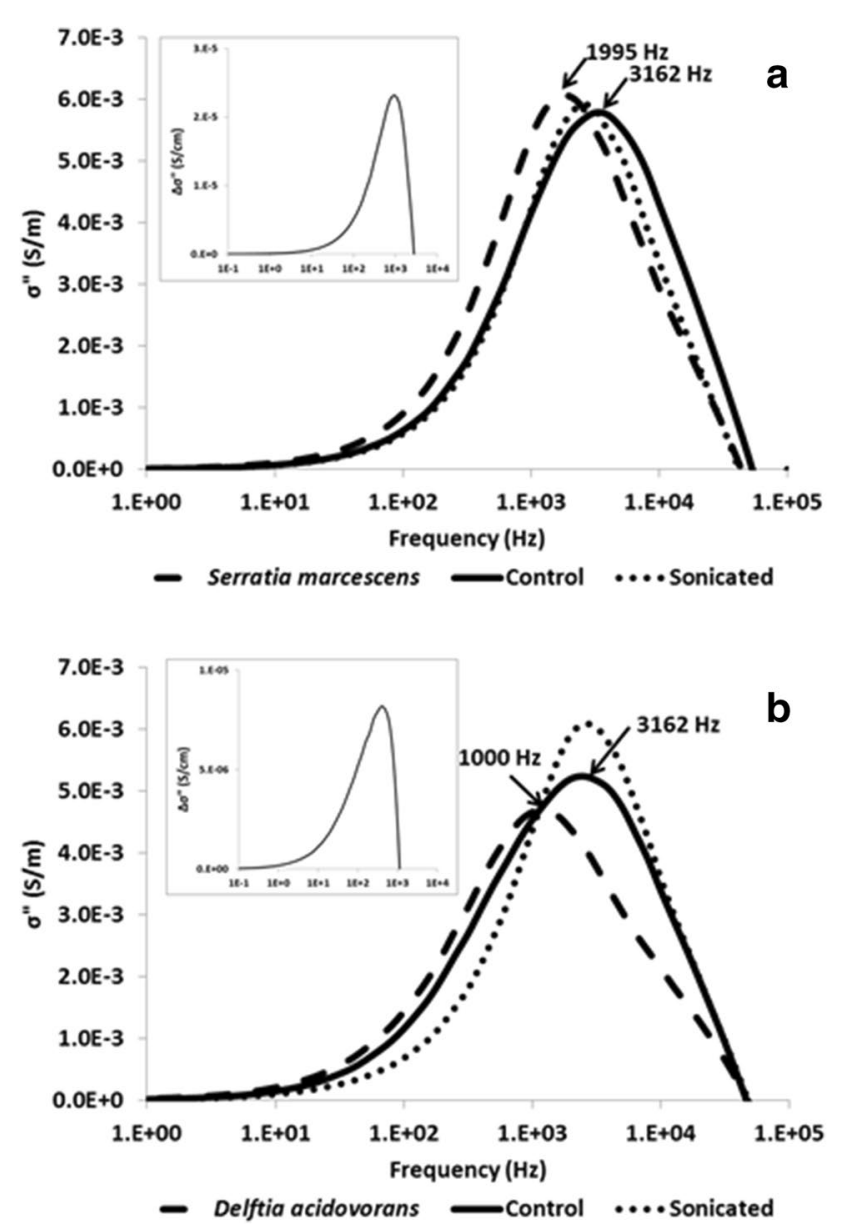

Fig. 7 Imaginary conductivity $\left(\sigma^{\prime \prime}\right)$ of two pure bacterial cultures grown on Al coupons, Al coupons alone and the coupons after biofilms were removed through sonication. Relative difference $\left(\Delta \sigma^{\prime \prime}\right)$ between control and biofilm, below $2 \mathrm{kHz}$, is depicted in the inset

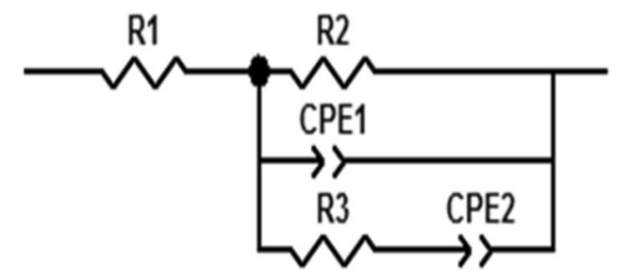

Fig. 8 Equivalent circuit used to fit the data where R1 represents the electrolyte resistance at high frequency and CPE1 and R2 represent a constant phase element and charge transfer resistance, respectively, at lower to medium frequencies, with CPE2 and R3 representing a constant phase element and a modification of the charge transfer resistance, respectively, at low to high frequencies
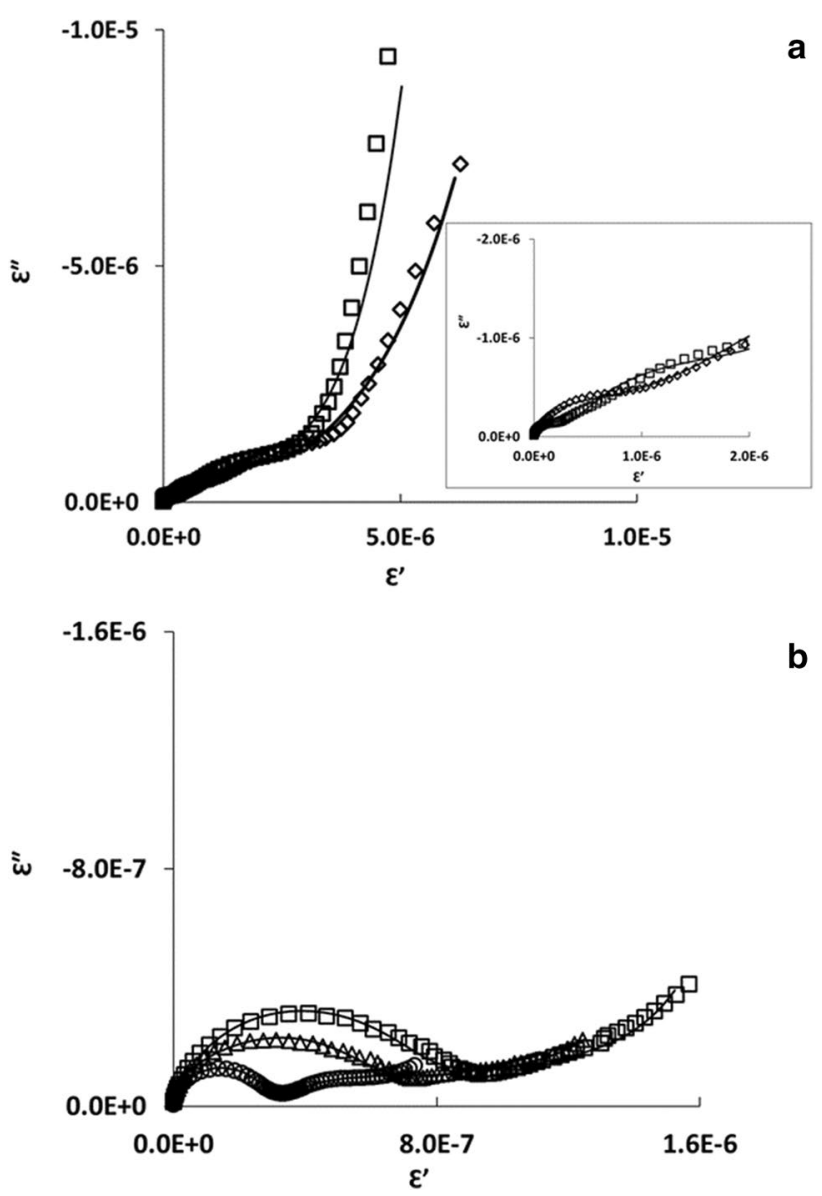

Fig. 9 Cole-Cole plots of surfaces with and without biofilms of pure bacterial cultures with (a) biofilms on glass $(0)$ compared to sterile glass $(\square)$ and Al coupons (b), with biofilms of $S$. marcescens $(O), D$. acidovorans $(\triangle)$ and sterile controls $(\square)$. Fit lines are depicted as lighter lines corresponding to specific treatments

acidovorans demonstrating the greatest change in $I_{0}$. D. acidovorans and $S$. marcescens biofilms were also associated with a decrease in $E_{\text {ocp }}$ (Fig. 10 and Table 1). 


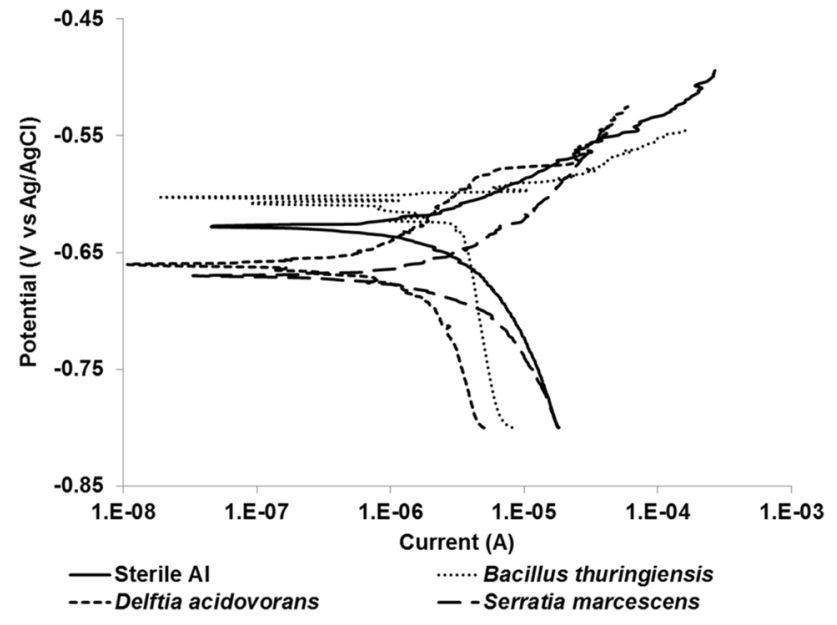

Fig. 10 Potentiodynamic polarization curves of Al coupons following 6 weeks of biofilm growth. Biofilms were removed through sonication and compared to sonicated sterile controls

\section{Discussion}

Evaluation of biofilms in many environments presents numerous logistical and technical challenges due to the location of the biofilms and/or their existence under harsh conditions such as high levels of ionizing radiation. Here, we demonstrated a non-optimized, electrochemical approach that provides a potential technique for remote, in situ, non-contact monitoring of biofilms growing on electrically conductive or inert surfaces. Surfaces evaluated in this study were glass and aluminum 1100. This aluminum alloy is used in parts of the nuclear industry. Glass was used to demonstrate the principle of non-contact biofilm analysis on surfaces, especially since glass surfaces would not produce corrosive by-products normally associated with metal biocorrosion processes. Therefore, changes in the EIS response on glass would be related solely to biofilm growth.

S. oneidensis MR-1 produces thick, three-dimensional biofilms when grown aerobically [25], and in the present study biofilms on glass demonstrated decreased impedance (IZI) below $2 \mathrm{kHz}$ compared to glass alone. This was directly related to the presence of biofilms, especially the production of extracellular polymeric substances (EPS). EPS are used by microbes to adhere to surfaces and also impart conductive properties [26-28]. Increased conductivity from EPS likely played a role in the present study by decreasing impedance (IZI) at the lower frequencies.

S. oneidensis MR-1 biofilms also demonstrated increased $\Delta \varphi$ with a peak around $1 \mathrm{kHz}$, indicative of a biofilm and consistent with previous studies. For instance, the buildup of an organic film on mild steel [29] as well as biofilms on stainless steel [30] and mild steel [31] was detected in the change in the phase angle shift as a function of film thickness. The membrane lipid bilayer of microbial cells allows the cells to interact electrochemically [32], and when evaluated with EIS, lipid membranes and peptides contribute to a phase shift $[14,15]$.

Microbial adhesion to surfaces, biofilm thickness and increased cell density all affect $\sigma^{\prime \prime}$ values, with a strong bias to living cells [12]. Abdel Aal et al. [11] defined $\sigma^{\prime \prime}$ to represent the polarization (energy storage) term and demonstrates increased $\sigma^{\prime \prime}$ values during microbial growth studies. The present study demonstrated that Shewanella biofilms increased the magnitude of $\sigma^{\prime \prime}$ relative to controls in the frequency range of about $10 \mathrm{~Hz}$ to $1 \mathrm{kHz}$. The biofilms also caused a $>1 \mathrm{kHz}$ shift in the $\sigma^{\prime \prime}$ peak from 2510 to $1260 \mathrm{~Hz}$. This peak shift could be due to chemical bonds associated with microbial adhesion. Changes in imaginary conductivity $\left(\Delta \sigma^{\prime \prime}\right)$ are associated with increased microbial biomass [11-13] and were detected in these studies as well.

The same technique as above was also used to monitor biofilms grown on aluminum coupons and produced similar results. The cultures isolated from nuclear cooling basins all grew on Al coupons with $S$. marcescens and $D$. acidovorans, demonstrating the most biofilm production and the strongest EIS responses. These species have previously been shown to cause biocorrosion on metal surfaces as biofilms [33, 34]. Responses to EIS analysis by $B$. thuringiensis were minimal, likely due to the low bacterial densities of biofilms in these studies. Overall, the greater the cell density (protein content), the more pronounced were the EIS responses, indicating that this technique offers potential for non-contact evaluation of biofilms.

Similar to S. oneidensis MR-1 biofilms on glass, S. marcescens and $D$. acidovorans biofilms on aluminum demonstrated decreased impedance. Decreased IZI also resulted from biofilms grown on stainless steel [26]. S. marcescens and $D$. acidovorans biofilms also demonstrated increased $\Delta \varphi$ at about $1 \mathrm{kHz}$, increased $\Delta \sigma^{\prime \prime}$ (below $1 \mathrm{kHz}$ ) and a shift in $\sigma^{\prime \prime}$ peak height by more than $1 \mathrm{kHz}$. The $\sigma^{\prime \prime}$ peak shifted back to higher frequencies after removal of the biofilms via sonication, indicating that the presence of the biofilms contributed to this phenomenon. The shift in the peak of $\sigma^{\prime \prime}$ is of interest and may be a result of surface interactions by the biofilm. The $\sigma$ " peak for sonicated coupons that had D. acidovorans biofilms were higher and narrower, indicating that the coupon surface changed as a result of biofilm sonication.

The influence of microbial activity on dielectric permittivity and double-layer capacitance was demonstrated here with biofilms on glass and aluminum. At the higher frequencies, biofilms on glass demonstrated greater double-layer capacitance than that of clean, sterile glass. Living bacterial cells increase permittivity values due to cell 
density, cell surface charge and metabolic activity [16] as was evident here, especially since glass is electrochemically inert. Low-frequency data of the biofilm on glass corresponded to the diffusion of the signal through the biofilm as compared to the limited diffusion exhibited by the glass. A decrease in double-layer capacitance was detected with mature biofilms on aluminum coupons relative to the sterile coupons. The decrease in this case corresponds to other studies of biofilms on conductive surfaces, such as metals, and relates to the biofilm density decreasing the overall double-layer capacitance, relative to surface properties without a biofilm.

The increase in $\Delta \varphi$ at the lower frequency range could indicate the presence of $\mathrm{Al}$ oxides as previously described [24] and correlates with biofilm density and biofilm-associated Al dissolution. The potentiodynamic analysis also demonstrated biofilm-associated changes in the surface and corrosion behavior of the Al coupons. Although $B$. thuringiensis had a higher percentage of $\mathrm{Al}$ associated with biomass, the low biomass density likely contributed to the minimal change in phase shift from 1 and $0.01 \mathrm{~Hz}$.

Interactions between bacterial biofilms and surfaces are complex and dynamic. Here, we demonstrated a novel technique that can be used to study the electrochemical dynamics of biofilms without physically damaging the biofilm through sampling. One advantage is the ability to more easily study biofilms on inert surfaces. More complex studies can be performed with this technique, such as the electrochemical behavior of bacterial growth dynamics relative to biofilm growth and the role of diffusion as it relates to biofilm dynamics. This approach to biofilm analysis also provides opportunities to study biofilms in situ, in real time.

\section{Conclusions}

Previous EIS evaluations of biofilms were restricted to electrochemically conductive surfaces, where the biofilmcontaining surface was the working electrode. Here, we demonstrate a non-contact technique used to evaluate surfaces for biofilms related to MIC. Potential advantages to this approach include a technique that does not physically disturb a biofilm and a way to monitor biofilms on electrochemically inert surfaces as well as metal surfaces.

Acknowledgements This research was supported by the Savannah River National Laboratory through the Laboratory Directed Research and Development Program; Strategic Initiative. This document was prepared in conjunction with work accomplished at SRNL under Contract No. DE-AC09-08SR22470 with the U.S. Department of Energy.
The authors would like to acknowledge the excellent technical assistance of the late Charles E. Milliken. The authors would also like to thank Dr. Sirivatch Shimpalee for valuable discussions about the manuscript.

\section{Compliance with ethical standards}

Conflict of interest The authors have no financial and/or personal relationships with other people or organizations that could inappropriately influence (bias) their work.

Open Access This article is licensed under a Creative Commons Attribution 4.0 International License, which permits use, sharing, adaptation, distribution and reproduction in any medium or format, as long as you give appropriate credit to the original author(s) and the source, provide a link to the Creative Commons licence, and indicate if changes were made. The images or other third party material in this article are included in the article's Creative Commons licence, unless indicated otherwise in a credit line to the material. If material is not included in the article's Creative Commons licence and your intended use is not permitted by statutory regulation or exceeds the permitted use, you will need to obtain permission directly from the copyright holder. To view a copy of this licence, visit http://creativecommons org/licenses/by/4.0/.

\section{References}

1. Videla HA, Herrera LK (2005) Microbially influenced corrosion: looking to the future. Int Microbiol 8:169-180

2. Trif L, Shaban A, Telegdi J (2018) Electrochemical and surface analytical techniques applied to the investigation of MIC. Corros Rev 34:1e15. https://doi.org/10.1515/corrrev-2017-0032

3. Tanaka N, Kogo T, Hirai N, Ogawa A, Kanematsu H, Takahara J, Awazu A, Fujita N, Haruzono Y, Ichida S, Tanaka Y (2019) In-situ detection based on the biofilm hydrophilicity for environmental biofilm formation. Sci Rep 9:8070. https://doi.org/10.1038/s4159 8-019-44167-6

4. Bhattacharyya R (2014) Interim storage of spent nuclear fuel: an assessment of technologies and challenges. J Nucl Eng Technol 64:1-11

5. Wolfram JH, Dirk WJ (1997) Biofilm development and the survival of microorganisms in water systems of nuclear reactors and spent fuel pools. In: Wolfram JH (ed) Microbial degradation processes in radioactive waste repository and in nuclear fuel storage areas. Kluwer Academic Pub, Dordrecht, pp 139-147

6. Louthan MR Jr (1997) The potential for microbiologically influenced corrosion in the Savannah River spent fuel storage pools. In: Wolfram JH (ed) Microbial degradation processes in radioactive waste repository and in nuclear fuel storage areas. Kluwer Academic Pub, Dordrecht, pp 131-137

7. Santo Domingo JW, Berry CJ, Summer M, Fliermans CB (1998) Microbiology of spent nuclear fuel storage basins. Curr Microbiol 37:387-394

8. Bruhn DF, Frank SM, Roberto FF, Pinhero PJ, Johnson SG (2009) Microbial biofilm growth on irradiated, spent nuclear fuel cladding. J Nucl Mater 384:140-145

9. Bagwell CE, Noble PA, Milliken CE, Li D, Kaplan DI (2018) Amplicon sequencing reveals microbiological signatures in spent nuclear fuel storage basins. Front Microbiol 9:377. https://doi. org/10.3389/fmicb.2018.00377 
10. Lehman RM, Colwell FS, Bala GA (2001) Attached and unattached microbial communities in a simulated basalt aquifer under fracture- and porous-flow conditions. Appl Environ Microbiol 67:2799-2809

11. Abdel Aal GZ, Atekwana AE, Rossbach S, Werkema DD (2010) Sensitivity of geochemical measurements to the presence of bacteria in porous media. J Geophys Res 115:G03017

12. Abdel Aal GZ, Atekwana EA, Atekwana EA (2010) Effect of bioclogging in porous media on complex conductivity signatures. J Geophys Res 115:G00G07

13. Ntarlagiannis D, Ferguson A (2008) SIP response of artificial biofilms. Geophysics 74:A1-A5

14. Laputkova G, Legin M, Sabo J (2005) Impedance of agar-supported bilayer membranes modified with alamenthicin. Scr Med $74: 227-234$

15. Salamon Z, Lindblom G, Tollin G (2003) Plasmon-waveguide resonance and impedance spectroscopy studies of the interaction between penetratin and supported lipid bilayer membranes. Biophys J 84:1796-1807

16. Zhang C, Slater L, Prodan C (2013) Complex dielectric properties of sulfate-reducing bacteria suspensions. Geomicrobiol J 30:490-496

17. Prodan C, Mayo F, Claycomb JR, Miller JH Jr, Benedik MJ (2004) Low-frequency, low-field dielectric spectroscopy of living cell suspensions. J Appl Phys 95:3754-3756

18. Poortinga AT, Bos R, Norde W, Busscher HJ (2002) Electric double layer interactions in bacterial adhesion to surfaces. Surf Sci Rep 47:1-32

19. Kim T, Kang J, Lee J-H, Yoon J (2011) Influence of attached bacteria and biofilm on double-layer capacitance during biofilm monitoring by electrochemical impedance spectroscopy. Water Res 45:4615-4622

20. Kim S, Yu G, Kim T, Shin K, Yoon J (2012) Rapid bacterial detection with an integrated array electrode by electrodhemical impedance spectrometry. Electrochim Acta 82:126-131

21. van Westing EPM, Ferrari GM, Geenen FM, de Wit JHW (1993) In situ determination of the loss of adhesion of barrier epoxy coatings using electrochemical impedance spectroscopy. Prog Org Coat 23:89-103

22. Marsili E, Rollefson JB, Baron DB, Hozalski RM, Bond DR (2008) Microbial biofilm voltammetry: direct electrochemical characterization of catalytic electrode-attached biofilms. Appl Environ Microbiol 74:7329-7337

23. Hobbie JE, Daley RJ, Jasper S (1977) Use of Nucleopore filters for counting bacteria by fluorescence microscopy. Appl Environ Microbiol 33:1225-1228
24. Hitzig J, Juttner K, Lorenz WJ, Paatsch W (1986) AC-impedance measurements on corroded porous aluminum oxide films. J Electrochem Soc 133:887-892

25. McLean JS, Majors PD, Reardon CL, Bilskis CL, Reed SB, Romine $M F$, Fredrickson JK (2008) Investigations of structure and metabolism within Shewanella oneidensis MR-1 biofilms. J Microbiol Methods 74:47-56

26. Cao B, Shi L, Brown RN, Xiong Y, Fredrickson JK, Romine MF, Marshall MJ, Lipton MS, Beyenal H (2011) Extracellular polymeric substances from Shewanella sp. HRCR-1 biofilms: characterization by infrared spectroscopy and proteomics. Environ Microbiol 13:1018-1031

27. Finkenstadt VL (2005) Natural polysaccharides as electroactive polymers. Appl Microbiol Biotechnol 67:735-745

28. Turick CE, Beliaev A, Ekechukwu AA, Poppy T, Maloney A, Lowy DA (2009) The role of 4-hydroxyphenylpyruvate dioxygenase in enhancement of solid-phase electron transfer by Shewanella oneidensis MR-1. FEMS Microbiol Ecol 68:223-235

29. Tan YJ, Bailey S, Kinsella B (1996) An investigation of the formation and destruction of corrosion inhibitor films using electrochemical impedance spectroscopy (EIS). Corros Sci 38:1545-1561

30. Cheng S, Tian J, Chen S, Lei Y, Chang X, Liu T, Yin Y (2009) Microbially influenced corrosion of stainless steel by marine bacterium Vibrio natriegens: (I) Corrosion behavior. Mater Sci Eng C 29:751-755

31. Chongdar S, Gunasekaran G, Kumar P (2005) Corrosion inhibition of mild steel by anaerobic biofilm. Electrochim Acta 50:4655-4665

32. Markx GH, Davey CL (1999) The dielectric properties of biological cells at radiofrequencies: applications in biotechnology. Enzyme Microb Technol 25:161-171

33. Rajasekar A, Ting Y-P (2010) Microbial corrosion of aluminum 2024 aeronautical alloy by hydrocarbon degrading bacteria Bacillus cereus ACE4 and Serratia marcescens ACE2. Ind Eng Chem Res 49:6054-6061

34. San NO, Nazir H, Donmez G (2012) Microbiologically influenced corrosion of NiZn Alloy coatings by Delftia acidovorans bacterium. Corros Sci 64:198-203

Publisher's Note Springer Nature remains neutral with regard to jurisdictional claims in published maps and institutional affiliations. 